IdeAs

Idées d'Amériques

15 | 2020

Eau et gestion de l'eau dans les Amériques

\title{
Las desigualdades territoriales y el 18-0 de Chile: algunos antecedentes
}

Rodrigo Marquez y José Viacava

\section{OpenEdition}

\section{Journals}

Edición electrónica

URL: http://journals.openedition.org/ideas/8404

DOI: 10.4000/ideas.8404

ISSN: 1950-5701

Editor

Institut des Amériques

Referencia electrónica

Rodrigo Marquez y José Viacava, «Las desigualdades territoriales y el 18-0 de Chile: algunos antecedentes », IdeAs [En línea], 15 | 2020, Publicado el 01 marzo 2020, consultado el 25 marzo 2020 URL : http://journals.openedition.org/ideas/8404 ; DOI : https://doi.org/10.4000/ideas.8404

Este documento fue generado automáticamente el 25 marzo 2020.

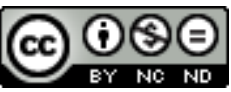

IdeAs - Idées d'Amériques est mis à disposition selon les termes de la licence Creative Commons Attribution - Pas d'Utilisation Commerciale - Pas de Modification 4.0 International. 


\title{
Las desigualdades territoriales y el 18-O de Chile: algunos antecedentes
}

\author{
Rodrigo Marquez y José Viacava
}

1 Para algunos pudiera llamar la atención que un pequeño país de Sudamérica, con una población no superior a los 18 millones de habitantes, con un ingreso per cápita de U\$26.317 (International Monetary Fund, 2019), exhibiendo un nivel de pobreza por ingresos del 8,6\% (Ministerio de Desarrollo Social, 2018), con el más alto nivel de desarrollo humano de américa latina (PNUD 2019) y con un significativo acceso a bienes de consumo se transformara en escenario de expresión de un profundo y generalizado malestar social ${ }^{1}$. Para otros, sin embargo, lo que llama la atención es que dicho malestar haya tardado tanto en expresarse en protestas como las observadas recientemente dado que, por décadas, junto a esos logros, Chile exhibió las más altas cifras de desigualdad entre los países de la OCDE incluso superiores a las de varios países de América Latina ${ }^{2}$.

2 A otros les sorprende que este estallido social se haya manifestado no sólo en las grandes ciudades donde se concentra la población si no que se haya expandido muy rápidamente y expresado en casi todas las ciudades, pueblos, localidades, aldeas y caseríos del país sin importar su tamaño o su distancia respecto del centro demográfico y político, Santiago. No es menor recordar que el gatillante inmediato de las primeras acciones de protesta - el alza del precio del boleto del metro o tren subterráneo (ver artículo de Larrouqué en este dossier) es una cuestión de exclusivo interés de la capital - que concentra más de la mitad de la población total del país. Y sin embargo, junto con un cúmulo de otras situaciones de fondo, todo Chile se unió a las protestas.

3 Y es que la desigualdad de Chile tiene una importante dimensión territorial. El país es reconocido como uno de los miembros de la OCDE que, a pesar de sus avances en indicadores objetivos, muestra aún las mayores brechas territoriales en la distribución de los beneficios y los perjuicios del desarrollo (PNUD, 2018).

4 Evidentemente esta situación no ha sido casual o azarosa. Es producto de un modelo económico-productivo neoliberal (ver artículo de Gárate en este dossier), con un énfasis extractivo y primario donde el territorio y sus recursos son observados como un stock 
más que un medio finito como alternativa de bienestar social. Hay que precisar tambien que Chile ha optado por un modelo político altamente centralista desde el inicio de la República.

\section{La desigualdad como "renuncia" del Estado}

5 Durante décadas - particularmente agudizados en el último cuarto del siglo $\mathrm{xx}$-, la planificación y programación pública en la conducción del desarrollo quedó relegada a un segundo plano, cediéndole al mercado las capacidades de explotación de los medios disponibles para ello. Esta "renuncia" del Estado a emplear sus capacidades para orientar estratégicamente el progreso armónico de los territorios, a favor de las fuerzas del mercado, quedó reflejada en un informe de la OCDE donde se estableció que, a pesar del crecimiento sostenido experimentado por la economía del país, los territorios no habían sido capaces de alcanzar grados de desarrollo homogéneos entre sí (OCDE, 2009). Tal es así, que en el informe realizado por la OCDE en el 2017, se indica que Chile se mantiene como el país más desigual de todos $(0,46 \%)$ en comparación con el resto de los países de la organización, "manteniendo un sistema tributario que favorece a los más ricos, reduciendo las posibilidades de movilidad social".

Esta desigualdad no sólo se constituyó en un factor que atentó contra su propio desarrollo: también afectó la relación entre la dimensión Economía-Sociedad CivilPolítica provocando un abismo entre estas dos últimas, tal como se ha evidenciado desde octubre del 2019 hasta hoy.

7 Como se observará a continuación, los ciudadanos/as de los territorios alejados de las áreas urbanas y metropolitanas del país tienen la percepción que no accederán a las mismas oportunidades de incidencia ni participarán, incluso, de los mecanismos de representación de intereses ante sus autoridades que chilenos/as de otras regiones y comunas del país.

8 Una reciente encuesta efectuada a los habitantes de distintas regiones en Chile (Barómetro Regional, 2019), evidenció la necesidad que sus "autoridades tomen más en cuenta las opiniones de la gente" a nivel regional. Es curioso: cuando comparativamente la literatura nos indica que la cercanía de las autoridades y sus ciudadanos/as conviven mejor a niveles subnacionales, este resultado nos muestra la existencia de una brecha importante. De este modo, la "renuncia" del Estado a su rol como agente del desarrollo se ha sumado también a su rol articulador entre los ciudadanos y los mecanismos de representación y participación política en el territorio.

\section{La desigualdad territorial vista desde las personas}

9 Con todo, la experiencia de la desigualdad territorial que viven chilenos y chilenas no admite visiones simplistas: ésta tiene una complejidad que no parece saltar a primera vista, pero que es muy relevante atender si se quiere sintonizar con el verdadero reclamo ciudadano en relación con lo que ocurre en los territorios. Dicha complejidad se expresa, por un lado, en los aspectos objetivos del fenómeno y también, por otro lado, en la evaluación subjetiva que se tiene de dicha desigualdad territorial.

En el primer aspecto, los datos objetivos, apreciamos que junto con avances en materia de mayores oportunidades y capacidades en los diversos territorios del país (es decir la 
convergencia territorial en indicadores básicos del desarrollo), persisten bolsones de desigualdad en territorios específicos y se levantan nuevas expresiones de la misma al observarse cuestiones de nuevo tipo (por ejemplo el impacto del cambio climático) o tradicionales, pero que se complejizan (por ejemplo la demanda por atención médica especializada). Todas estas situaciones afectan la vida de las personas de manera relevante y diferenciada según el lugar donde habitan.

11 Por otro lado, desde la perspectiva de las percepciones, es decir, la manera en que las personas evalúan sus vidas y su trayectoria personal y familiar, se aprecia que, pese a los avances objetivos, la experiencia de desigualdad sigue presente y ampliamente constatada en estudios de opinión sobre desarrollo humano en Chile (por ejemplo ver PNUD 2018 o PNUD 2015). Esta crítica tiene sus particularidades. Por ejemplo, el diferencial de oportunidades en las diversas regiones de Chile versus la gran capital en Santiago es una opinión que surge de manera nítida. Al parecer las personas resienten que pese a los avances que pueden observarse, sigue aún existiendo la imagen de un "otro lugar" donde se concentrarían las mejores y más potentes oportunidades: Santiago. Este espacio en consecuencia condensa la crítica respecto de la injusticia de esa distribución de las mejores oportunidades, cuestión que cada día se hace menos tolerable en la opinión pública. Una crítica similar aparece ahora pensando "hacia dentro" del propio territorio como un reclamo al "centralismo intra regional" que se expresa en la menor atención que parece dársele a las localidades más alejadas de las capitales regionales. Se reproduciría el fenómeno de la desigualdad territorial, pero ahora desde la perspectiva del ejercicio del poder.

12 Veamos algunos datos que fundan las conclusiones anteriores: una encuesta sobre Desarrollo Humano y territorio del año 2016 indagó en el nivel de desigualdad de oportunidades entre las distintas regiones del país que perciben los chilenos y chilenas $^{3}$. Tal como se aprecia en el siguiente gráfico, la amplia mayoría de los encuestados considera que las oportunidades entre las distintas regiones son bastante $\mathrm{o}$ muy desiguales $(66,8 \%)^{4}$.

Gráfico 1. Si tuviera que evaluar la desigualdad entre las regiones de Chile, usted diría que las oportunidades que existen entre las regiones son: (porcentaje)

\section{(c) Encuesta de Desarrollo Humano, PNUD, 2016.}

13 La percepción de desigualdad es especialmente aguda cuando quienes residen en regiones comparan sus oportunidades con aquellas a las que es posible acceder en Santiago. Tal como se observa en la siguiente tabla, un $68 \%$ de quienes no residen en la Región Metropolitana ( $\mathrm{RG}$ ), considera que en el lugar donde vive hay menos oportunidades que en la capital (Tabla 1).

Tabla 1. Pregunta sobre oportunidades que presenta el lugar donde vive comparando regiones con Santiago.

\begin{tabular}{|l|l|l|}
\hline & $\begin{array}{l}\text { Personas de regiones } \\
\text { comparando con Santiago }\end{array}$ & $\begin{array}{l}\text { Personas de Santiago comparando } \\
\text { con otras regiones }\end{array}$ \\
\hline $\begin{array}{l}\text { Más oportunidades } \\
\text { donde vive }\end{array}$ & 12,9 & 32,0 \\
\hline
\end{tabular}




\begin{tabular}{|l|l|l|}
\hline $\begin{array}{l}\text { Menos oportunidades } \\
\text { donde vive }\end{array}$ & 67,6 & 20,8 \\
\hline $\begin{array}{l}\text { Las mismas } \\
\text { oportunidades }\end{array}$ & 16,9 & 43,4 \\
\hline NS/NR & 2,6 & 3,7 \\
\hline
\end{tabular}

(c) Encuesta de Desarrollo Humano, PNUD, 2016.

14 Al consultar acerca de la evolución en el tiempo de la desigualdad territorial, la encuesta sobre desarrollo humano y territorio muestra que un $43 \%$ de los encuestados considera que las desigualdades entre regiones han aumentado durante la última década. Un $28 \%$ cree que se han mantenido igual. Sólo un $24 \%$ piensa que han disminuido. Y respecto de los próximos diez años, un 39\% de la opinión pública encuestada cree que la desigualdad de oportunidades entre regiones de chile va a aumentar. Sólo un $26 \%$ piensa que disminuirá y un $28 \%$ piensa que se mantendrá igual.

En este punto se aprecia que la macrozona Norte (Arica a Coquimbo) existe una imagen más negativa de esta trayectoria: un $58 \%$ de los ahí encuestados piensa que las desigualdades entre regiones han venido aumentando y un $50 \%$ cree que seguirán aumentando en los próximos diez años. Por su parte, en la macro zona Sur (regiones desde Maule hasta Magallanes), se aprecia un aumento de porcentajes de respuesta que dan cuenta de aspectos positivos a esta trayectoria.

Tabla 2 Desigualdades de oportunidades

\begin{tabular}{|l|l|l|l|}
\hline \multicolumn{2}{|l|}{$\begin{array}{l}\text { Y si piensa en 10 años atrás, usted diría que } \\
\text { la desigualdad de oportunidades entre las } \\
\text { regiones de Chile... }\end{array}$} & $\begin{array}{l}\text { Y pensando en los próximos 10 años, usted } \\
\text { diría que la desigualdad de oportunidades } \\
\text { entre las regiones de Chile... }\end{array}$ \\
\hline Han aumentado & $43 \%$ & Va a aumentar & $39 \%$ \\
\hline Han disminuido & $24 \%$ & Va a disminuir & $26 \%$ \\
\hline $\begin{array}{l}\text { No han aumentado ni } \\
\text { disminuido }\end{array}$ & $28 \%$ & $\begin{array}{l}\text { No va a aumentar ni a } \\
\text { disminuir }\end{array}$ & $28 \%$ \\
\hline ns- nr & $5 \%$ & ns-nr & $5 \%$ \\
\hline Total & $100 \%$ & Total & $100 \%$ \\
\hline
\end{tabular}

(c) Encuesta opinión pública DH Y territorio 2016

Tabla 3 la desigualdad de oportunidades entre regiones

\begin{tabular}{|l|l|l|l|l|}
\hline \multicolumn{2}{|l|}{ LA DESIGUALDAD DE OPORTUNIDADES ENTRE REGIONES } \\
\hline Comparado con 10 años atrás & Zona Norte & Zona Centro & Zona Sur & Región Metropolitana \\
\hline
\end{tabular}




\begin{tabular}{|l|l|l|l|l|}
\hline Han venido aumentando & $58 \%$ & $49 \%$ & $42 \%$ & $36 \%$ \\
\hline Han venido disminuyendo & $12 \%$ & $16 \%$ & $28 \%$ & $26 \%$ \\
\hline Se han mantenido igual & $24 \%$ & $27 \%$ & $26 \%$ & $32 \%$ \\
\hline Ns/nr & $6 \%$ & $8 \%$ & $4 \%$ & $5 \%$ \\
\hline Total & $100 \%$ & $100 \%$ & $100 \%$ & $100 \%$ \\
\hline & & & & \\
\hline En los próximos 10 años & Zona Norte & Zona Centro & Zona Sur & Región Metropolitana \\
\hline Aumentarán & $51 \%$ & $49 \%$ & $38 \%$ & $31 \%$ \\
\hline Disminuirán & $18 \%$ & $15 \%$ & $30 \%$ & $30 \%$ \\
\hline Se mantendrán igual & $24 \%$ & $26 \%$ & $25 \%$ & $31 \%$ \\
\hline Ns/nr & $7 \%$ & $10 \%$ & $7 \%$ & $8 \%$ \\
\hline Total & $100 \%$ & $100 \%$ & $100 \%$ & $100 \%$ \\
\hline
\end{tabular}

\section{Un importante contraste entre la opinión de las élites regionales versus la opinión pública regional}

Un contraste muy significativo se aprecia al comparar las respuestas de las elites regionales $^{5}$ (los que toman las decisiones y son influyentes en los territorios) con las respuestas de la población en general en cuanto a la percepción de la evolución de las desigualdades regionales. Mientras una parte no mayoritaria, pero importante de las elites regionales ven una mejoría en el nivel de desigualdad entre regiones, la opinión pública ve un importante nivel de incremento de la misma en los últimos diez años. De igual manera, en relación con el futuro de esta situación, mientras las elites regionales mayoritariamente piensan que la desigualdad entre Regiones va a disminuir en los próximos diez años, la opinión pública piensa, en una alta proporción, que va a aumentar (ver tabla 4).

17 Es sin duda difícil explicar qué puede estar detrás de estas opiniones tan diferentes (y no es este el lugar para hacerlo). Basta aquí mostrar estos datos para fundar otro rasgo que ha sido señalado como distintivo de las tensiones sociales de Chile de los últimos años y exacerbadas en los hechos de los últimos meses: la enorme distancia y desconexión entre las elites y la ciudadanía, tanto en sus percepciones como en sus ámbitos de acción y sus decisiones.

18 Esta distancia se traduce en los hechos en una mayor dificultad y legitimidad para conducir los procesos sociales aún a nivel de territorios específicos. Es una doble falla: 
las élites no logran identificar qué es lo importante para las personas y, segundo, no logran transmitir a la ciudadanía una imagen de futuro positiva que permita aquilatar los avances realizados. Si la tarea de toda elite es construir relatos para interpretar las trayectorias pasadas y futuras de una comunidad, habría aquí una importante tarea pendiente para las elites regionales de cara a hacer frente a la desigualdad territorial.

Tabla 4 Percepción de desigualdad de oportunidades

\begin{tabular}{|l|l|l|l|l|l|}
\hline \multicolumn{3}{|l|}{$\begin{array}{l}\text { Y si piensa en 10 años atrás, usted diría que } \\
\text { la desigualdad de oportunidades entre las } \\
\text { regiones de Chile... }\end{array}$} & $\begin{array}{l}\text { Y pensando en los próximos 10 años, usted } \\
\text { diría que la desigualdad de oportunidades } \\
\text { entre las regiones de Chile... }\end{array}$ \\
\hline & $\begin{array}{l}\text { Elite } \\
\text { Regional }\end{array}$ & $\begin{array}{l}\text { Opinión } \\
\text { pública }\end{array}$ & & $\begin{array}{l}\text { Elite } \\
\text { Regional }\end{array}$ & $\begin{array}{l}\text { Opinión } \\
\text { pública }\end{array}$ \\
\hline Han aumentado & $23 \%$ & $43 \%$ & Va a aumentar & 19 & 39 \\
\hline Han disminuido & $42 \%$ & $24 \%$ & Va a disminuir & 54 & 26 \\
\hline $\begin{array}{l}\text { No han aumentado } \\
\text { ni disminuido }\end{array}$ & $34 \%$ & $28 \%$ & $\begin{array}{l}\text { No va a aumentar } \\
\text { ni a disminuir }\end{array}$ & 22 & 28 \\
\hline ns - nr & $1 \%$ & $5 \%$ & ns - nr & $5 \%$ & $7 \%$ \\
\hline Total & $100 \%$ & $100 \%$ & Total & $100 \%$ & $100 \%$ \\
\hline
\end{tabular}

(c) Encuesta de Elite Regional, PNUD 2016-2017. Encuesta opinión pública 2016

19 Como muestran estos datos, para los chilenos y chilenas, la desigualdad territorial es una de las dimensiones en que se expresa también su reclamo por una sociedad más justa e integrada. Esta demanda, ya conocida en el 2016 fue otra de las expresiones de la ciudadanía a la que parece no habérsele dado suficiente importancia. La evidencia estaba ahí. No se le tomó el peso suficiente.

Lo que sabemos hasta ahora es que detrás de esta demanda por un desarrollo más equitativo, con mayor igualdad territorial no hay sólo demandas de orden material o de cuestiones meramente redistributivas; hay también un propósito de ser reconocido como un legítimo partícipe de la comunidad política que es Chile. Ser reconocido, no mirado en menos, no dejado atrás y ser validado como un actor con plena capacidad para involucrarse en el debate de los asuntos públicos que interesan a cualquier sociedad en un Estado democrático de derecho.

\section{BIBLIOGRAFÍA}

Banco Mundial, Índice Gini, 2017, https://urlz.fr/bItc, consultado el 09/03/2020 
Barómetro Regional, Chile visto por sus regiones, 2019, https://urlz.fr/bIte, consultado el $09 / 03 / 2020$

Biblioteca del Congreso Nacional, Presupuesto Año 2019, 2019, https://urlz.fr/bItj, consultado el $09 / 03 / 2020$

Borja, Jordi et al., Descentralización y democracia Gobiernos locales en América Latina, Santiago, CLASCO, 1989.

CNN Chile, « Nobel de Economía sobre el estallido social: "La sorpresa fue que el malestar tardara en manifestarse"», Santiago, CNN, 28 de enero 2020, https://urlz.fr/bItG

International Monetary Fund, Report for Selected Countries and Subjects, Washington DC, 2019, https://www.imf.org/external/pubs/ft/weo/2019/01/weodata/index.aspx, consultado el $11 / 03 / 2020$

INE, Ingreso laboral promedio mensual en Chile fue de $\$ 573.964$ en 2018, INE, 2019, https:// ellibero.cl/alerta/ine-ingreso-laboral-promedio-mensual-en-chile-fue-de-573-964-en-2018/, consultado el 11/03/2020

Ministerio de Desarrollo Social, Pobreza y distribución de ingresos: presentación de resultados, Santiago, MDS, 2018, https://static.emol.cl/emol50/documentos/archivos/ 2018/08/21/20180821112716.pdf, consultado el 11/03/2020

OECD, Territorial Reviews (Chile), Paris, OCDE, 2009, https://www.oecd.org/cfe/regional-policy/ oecdterritorialreviewschile.htm, consultado el 11/03/2020

PNUD, Informe Desarrollo Humano en Chile "Los tiempos de la politización”. Chile, Santiago, PNUD, 2015, https://www.oecd.org/cfe/regional-policy/oecdterritorialreviewschile.htm, consultado el $11 / 03 / 2020$

PNUD, Encuesta de Desarrollo Humano, PNUD, Santiago, 2016.

PNUD, Desigualdad Regional en Chile: Ingresos, Salud y Educación en perspectiva territorial, PNUD, Santiago, 2018.

PNUD, Informe Mundial sobre Desarrollo Humano, PNUD, 2019.

SERNAMEG, Femicidios 2019, Santiago, Sernameg, 2019, https://www.sernameg.gob.cl/? page_id=27084, consultado el 11/03/2020

Subsecretaria de Desarrollo Regional y Administrativo, Programa para el Desarrollo de Territorios Rezagados, Santiago, SDRA, 2014, https://urlz.fr/bIvb, consultado el 9/03/2020

Sitiglitz, Joseph, El precio de la desigualdad, Taurus, Buenos Aires, 2012.

\section{NOTAS}

1. Agradecemos la colaboración de Francisca Díaz V. en la confección de algunos datos y referencias bibliográficas.

2. Ver por ejemplo entrevista a Joseph Stiglitz, en BBC, reproducido en CNN Chile (2020) recuperado el 30/01/2020

3. Encuesta de 2200 casos, cara a cara, hecha en hogares a mayores de 18 años, con representatividad nacional, urbano rural y por macrozonas. Diseñó probabilístico trietápico. Error +- 3\%.

4. Este resultado en consistente con los hallazgos de la encuesta "Desiguales" (PNUD, 2016), donde un $64 \%$ de los encuestados en otras regiones y un 70\% de los encuestados en la Región 
Metropolitana considera que Chile es muy desigual entre regiones. (Valores 8, 9 y 10 de la escala entre 1 y 10)

5. Encuesta cualitativa a una muestra estructural de actores de las elites regionales de Chile. 250 casos provenientes de los ámbitos del poder político, económico, social y cultural. Casos validados por jueces externos. Encuesta realizada por la "Red de investigadores en Desarrollo Humano" 2016.2017.

\section{AUTORES}

RODRIGO MARQUEZ

Rodrigo Marquez : FLACSO-Chile, Universidad de Valparaiso rodrigo.marquez@flacsochile.org

JOSÉ VIACAVA

José Viacava : INAP-Universidad de Chile

jose.viacava@iap.uchile.cl 